\title{
Glowing in the dark: case report of a clue-poor melanoma unmasked by polarized dermatoscopy
}

\author{
Yoon K. Cohen ${ }^{1}$, David J. Elpern ${ }^{1}$, Deon Wolpowitz ${ }^{2}$, Cliff Rosendahl ${ }^{3}$
}

\begin{abstract}
1 The Skin Clinic, Williamstown, Massachusetts, USA
2 Department of Dermatology and Section of Dermatopathology, Boston University School of Medicine, Boston, Massachusetts, USA

3 School of Medicine, University of Queensland, Brisbane, Australia
\end{abstract}

Keywords: melanoma, nevus, congenital-type nevus, polarized dermoscopy, polarized dermatoscopy, dermatopathology, polarizing-specific white lines, chrysalis, BLINCK algorithm, chaos and clues algorithm

Citation: Cohen YK, Elpern DJ, Wolpowitz D, Rosendahl C. Glowing in the dark: case report of a clue-poor melanoma unmasked by polarized dermatoscopy. Dermatol Pract Concept. 2014;4(1):14. http://dx.doi.org/10.5826/dpc.0401a14

Received: September 15, 2013; Accepted: October 9, 2013; Published: January 31, 2014

Copyright: $@ 2014$ Cohen et al. This is an open-access article distributed under the terms of the Creative Commons Attribution License, which permits unrestricted use, distribution, and reproduction in any medium, provided the original author and source are credited.

Funding: None.

Competing interests: The authors have no conflicts of interest to disclose.

All authors have contributed significantly to this publication.

Corresponding author: Cliff Rosendahl, MBBS, Ph.D., PO Box 734, Capalaba, QLD 4157, Australia. Tel. +61 73245 3011; Fax. +61 7 3245 3022. Email: cliffrosendahl@bigpond.com

ABSTRACT We report a case of a melanoma arising in a congenital-type compound nevus, which was excised because it was observed by both the patient and the treating dermatologist to have changed. Because the lesion was routinely photo-documented with both polarized and non-polarized dermatoscopy images prior to excision, these images were available for subsequent examination. Matched images are presented in what appears to be unique in the published literature: polarizing-specific white lines are identified as a compelling clue to the diagnosis of melanoma in a lesion that contains no clues apparent in the non-polarized image. Dermatopathology images reveal that the melanoma is arising in conjunction with a congenital type nevus. As expected, dermatoscopic polarizing-specific white lines are evident on the melanoma but not the nevus, and while a possible explanation is discussed, this remains speculative.

\section{Case report}

A 27-year-old woman presented to a dermatology practice in Williamstown, Massachusetts with a pigmented skin lesion on her left anterior shoulder. She was originally seen four months earlier, and as the lesion was noted to be "somewhat atypical," she had been given a follow-up appointment to be reviewed in six months. She made the decision to present earlier because "the lesion had changed in color." On exami- nation a 4-5 $\mathrm{mm}$ diameter tan papule with an irregularity of pigment was observed and the notation was made in the patient's notes that " ... clinically this does not look very worrisome but the change is concerning ..." and for that reason excision biopsy was performed.

The preferred algorithmic method of the treating dermatologist (DJE) is a novel method known as 'BLINCK' [1]. This method combines both clinical and dermatoscopic clues to guide the decision to biopsy. The fact that the lesion was 


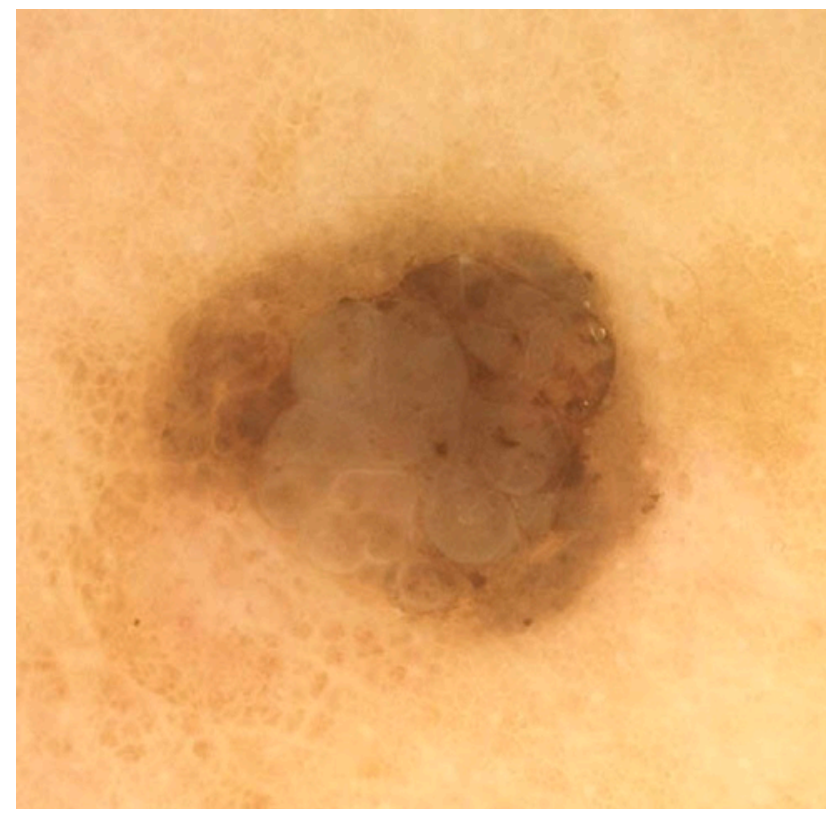

Figure 1. Non-polarized dermatoscopic image of a lesion on the anterior left shoulder of a 27-year-old woman. There is a pattern of apparently exophytic brown clods centrally, surrounded by a zone of structureless brown with a pattern of small brown clods peripherally. There are some eccentric foci of darker brown pigment on the right side of and to the right of the larger clods. [Copyright: @2014 Cohen et al.]

"lonely" (the only one of its kind in that location) and that the patient was "nervous" gave a score of two and that score leads to excision biopsy according to 'BLINCK'.

Prior to excision biopsy, dermatoscopic images were obtained in both polarizing and non-polarizing mode (Figures 1-3) with a Canfield DermScope (Canfield Scientific Inc. USA) coupled to an iPhone (Apple Inc. USA). The images

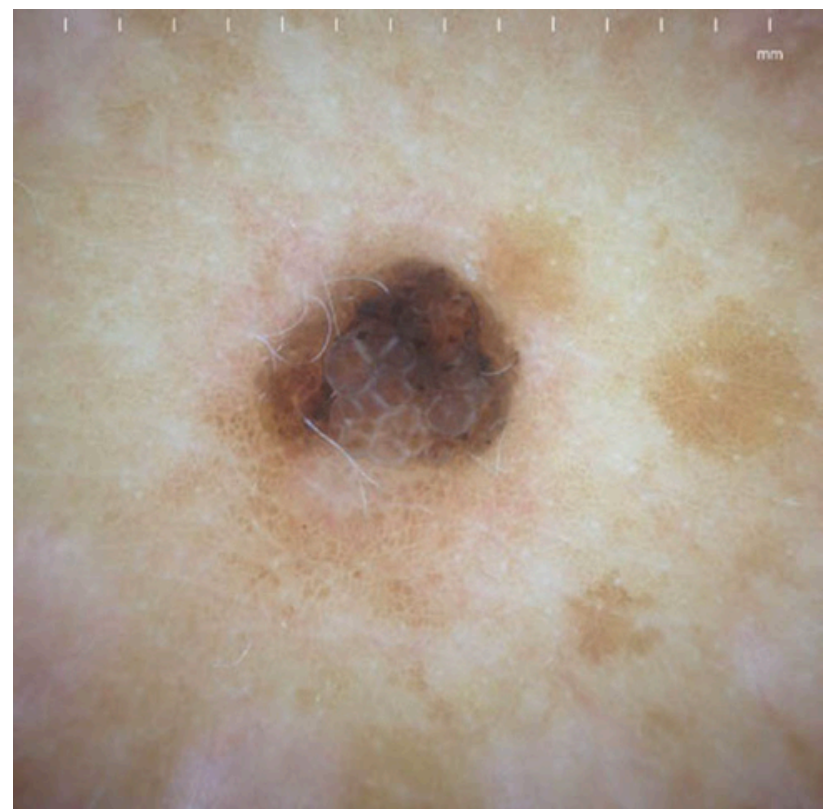

Figure 2. Polarized dermatoscopic image of the same lesion as displayed in Figure 1. In this image a millimeter scale reveals that the dimensions of the dark brown lesion are $5 \times 4 \mathrm{~mm}$. White lines that are clearly whiter than the patient's skin color are present over the center of the lesion and demarcate the clods seen with non-polarizing dermatoscopy (Figure 1). A pattern of small brown clods surrounds the lesion in some parts, extending to the borders of the image. Hairs are seen on the lesion peripheral to, but not over, the pattern of white lines. [Copyright: @2014 Cohen et al.]

were taken routinely as part of the clinical record of a lesion scheduled for excision biopsy to exclude melanoma, and this was done in spite of a low level of suspicion, which was based on the history of change rather than on the clinician's assessment of the dermatoscopic appearance.

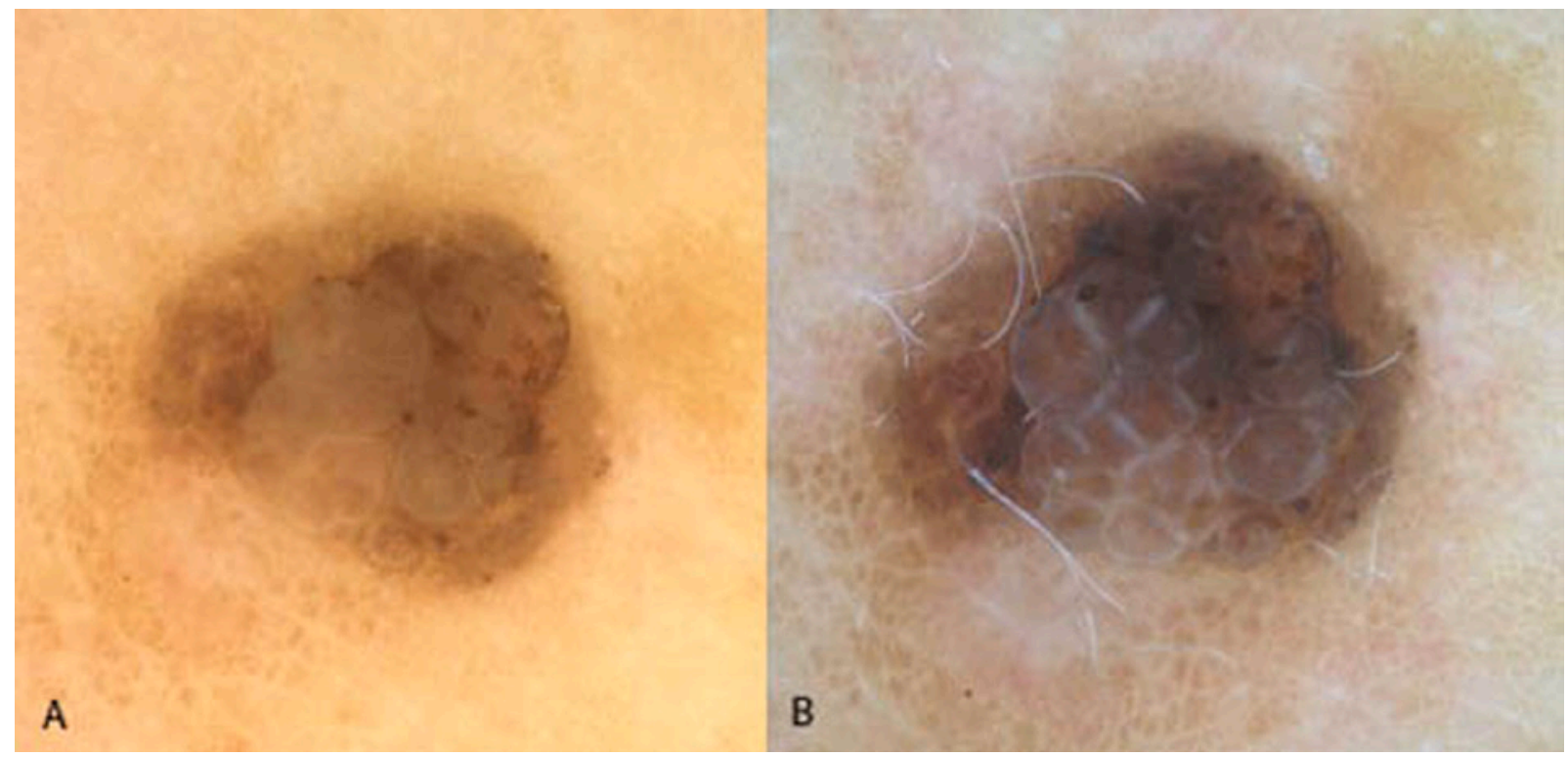

Figure 3. A couplet of (A) non-polarizing dermatoscopy and (B) polarizing dermatoscopy of the lesion displayed in Figures 1 and 2 revealing the correlation between the polarizing-specific white lines in B and the large brown clods in A. The smaller brown clods surrounding the lesion are not separated by white lines, but by skin-colored lines. Hairs are seen in image B, but they are not present on the central part of the lesion where the polarizing-specific white lines are seen. [Copyright: @2014 Cohen et al.] 


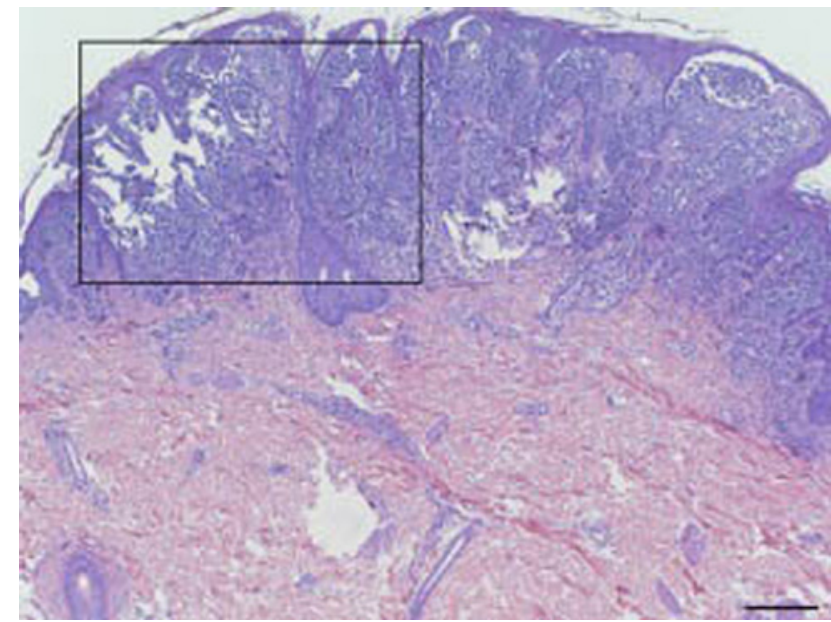

Figure 4. Low power hematoxylin and eosin image (40x) of the central exophytic portion of the lesion shown on Figures 1-3. A neoplastic proliferation of melanocytes is present in the epidermis as single cells and discohesive nests with clefting and in the dermis predominantly as nests. The boxed area is shown at higher power in Figure 5. Scale bar, $100 \mu \mathrm{M}$. [Copyright: @2014 Cohen et al.]

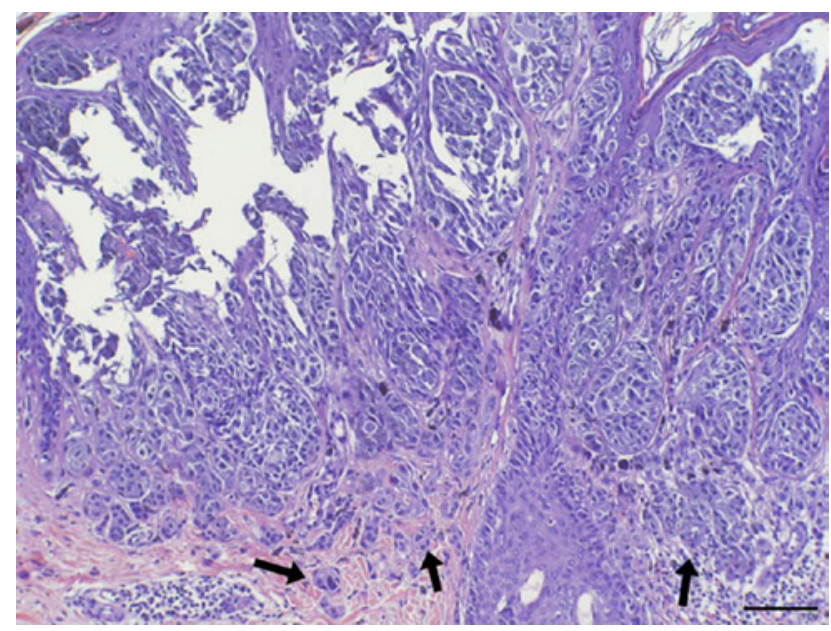

Figure 5. Hematoxylin and eosin image (100x) of the boxed area in Figure 4 showing severely atypical and epithelioid melanocytes distributed both in the epidermis as markedly discohesive and irregular junctional nests, as a near confluent proliferation of single cells along the dermoepidermal junction lining a hair follicle, and in the subjacent papillary dermis (black arrows). Fibrotic bands of collagen, many orientated vertically, are seen separating nests of neoplastic melanocytes. Scale bar, $100 \mu \mathrm{M}$. [Copyright: @2014 Cohen et al.]

Dermatopathologically (Figures 4-7) this lesion displayed both architectural and cytological criteria consistent with melanoma arising in conjunction with a compound melanocytic nevus with congenital nevus features, and benign nevus cells were reported as being present in the dermis at a deeper level than the deepest observed melanoma cell. The Breslow thickness was $1.01 \mathrm{~mm}$ and the mitotic rate was reported as 1 per square millimeter.

The lesion was re-excised with one a centimeter peripheral clearance margin, deep to but not including muscle

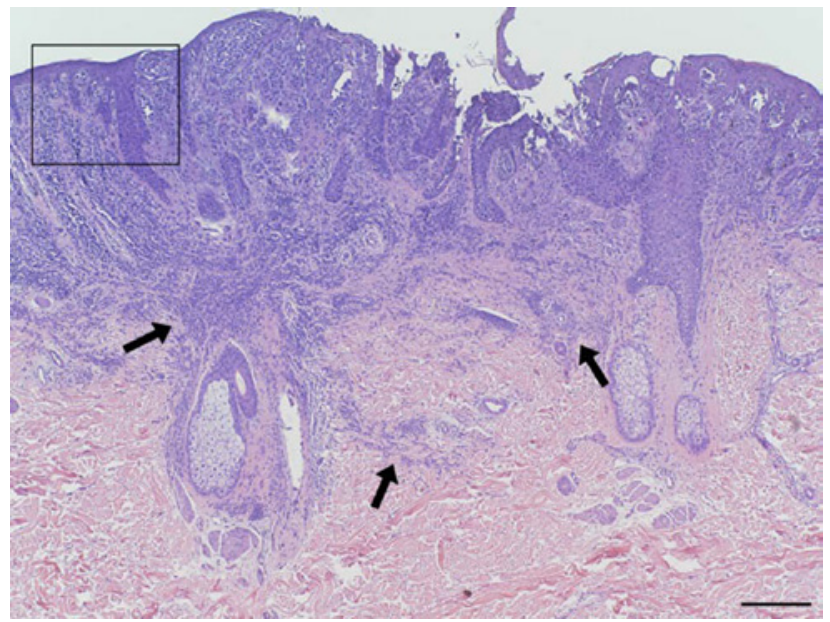

Figure 6. Hematoxylin and eosin image $(40 \mathrm{x})$ of part of the lesion shown in Figures 1-3 adjacent to the area shown in Figure 4. In addition to a neoplastic epidermal proliferation of melanocytes (boxed area upper left) there is a broad junctional and deep dermal melanocytic proliferation present with congenital nevus architecture. Arrowheads point to the dermal component. The boxed area is shown at higher power in figure 7. Scale bar, $100 \mu \mathrm{M}$. [Copyright: @2014 Cohen et al.]

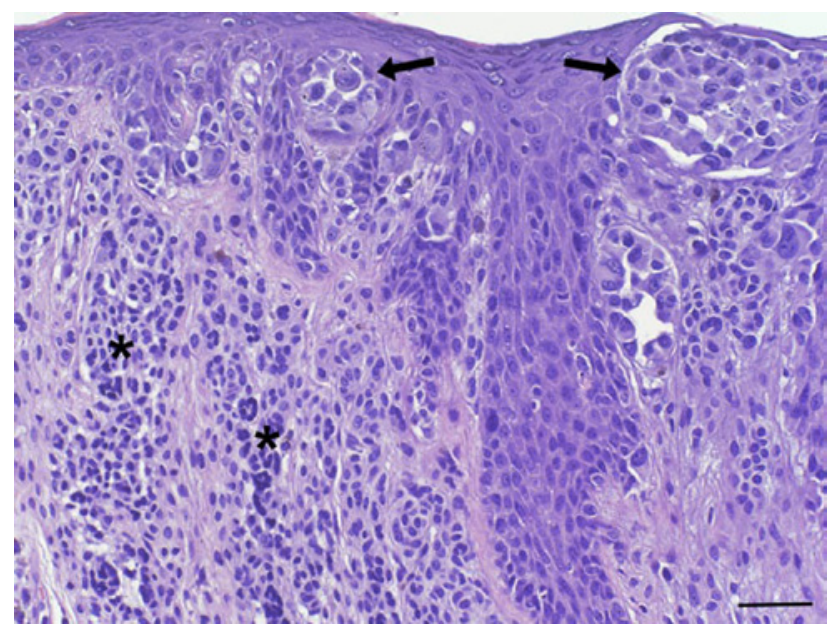

Figure 7. Hematoxylin and eosin image (200x) of the boxed area in Figure 6. Irregular junctional nests (black arrows) comprised of severely atypical epithelioid melanocytes overlie dermal nests of banal and nevic appearing melanocytes (asterisks). Scale bar, $100 \mu \mathrm{M}$. [Copyright: (02014 Cohen et al.]

fascia, and a sentinel lymph node biopsy was performed, which was negative.

\section{Conclusions}

Dermatoscopy relies on either fluid immersion or cross-polarization to reduce light scatter at the air-skin interface, and it is known that these different methods have an impact on the colors and structures which are displayed [2]. Non-polarized dermatoscopy has been said to provide superior rendition of 
superficial structures while polarized dermatoscopy reveals a clearer rendition of deeper structures as well as of structures not seen with non-polarized dermatoscopy, including four-dot clods (also known as rosettes) and polarizing-specific white lines (also known as chrysalis, crystalline structures and shiny white streaks) [3].

The most widely utilized and publicized method for recognizing melanomas based on their clinical appearance is the ABCD method [4]. This method relies on the lesion exhibiting asymmetry, an irregular border, color irregularity and a minimum diameter of $6 \mathrm{~mm}$. No clinical image was taken of the lesion reported here, but the dermatoscopy images reveal that it had asymmetry of color with two shades of brown, a regular border and dimensions of $5 \times 4 \mathrm{~mm}$, and therefore it would not have met the criteria for melanoma on the basis of the ABCD clinical algorithm.

The non-polarized dermatoscopic image of the lesion reported here (Figures 1 and 3A) shows a pattern of brown clods centrally surrounded by a zone of structureless brown, and further peripherally there is a pattern of small brown clods. There is some minor irregularity of pigment distribution, evident as several eccentric foci of dark brown color over the lighter brown clods.

The polarized image (Figures 2 and 3B) shows the same basic pattern as the non-polarized image, but in addition there is a pattern of white lines, whiter than surrounding skin color, and predominantly oriented in a perpendicular arrangement. Because these white lines are not present in the non-polarized dermatoscopy image, they can be described with certainty as dermatoscopic polarizing-specific white lines (PSWL). In the polarized images hairs are visible peripherally but they are absent over the central area where the polarizing-specific white lines are present (Figures 2 and 3B).

PSWL are a published clue to melanoma, Spitz nevus, basal cell carcinoma, dermatofibroma, scar tissue and benign lichenoid keratosis [5]. Although there is a large list of differential diagnoses for lesions with this clue, it is notable that if this clue is seen in a melanocytic lesion that is not a Spitz nevus, then the diagnosis of melanoma can be expected. It is also known that polarizing specific white lines are more frequently seen in melanomas, which are invasive [5]. The likelihood of Spitz nevus decreases with increasing age [6], and some authors recommend the excision of all Spitz nevi at any age due the fact that the distinction from melanoma can be challenging dermatopathologically [7]. In the largest study evaluating PSWL, of the non-biopsied lesions, none of 9750 Clark nevi, 90 congenital melanocytic nevi (CMN), 168 intradermal nevi (IDN) and 15 blue nevi displayed this structure, while of the biopsied lesions one of 20 Clark nevi, two of 10 IDN and none of one CMN displayed PSWL [5].

According to the original dermatoscopic method of classic pattern analysis [8], the global pattern of the lesion presented here is the so-called "cobblestone" pattern (pattern of clods) described as the pattern of a congenital type nevus and this lesion had none of the specific criteria of melanoma according to that method. Likewise this lesion did not score as a melanoma according to the ABCD dermatoscopic algorithm [9], the 3-point checklist [10], the 7-point checklist [11], the Menzies method [12] or the CASH algorithm [13]. The “Chaos \& Clues" algorithm [14,15] identifies suspicion for malignancy based on the presence of chaos (defined as asymmetry of structure and/or color) plus the presence of at least one of eight clues, including the clue of "white lines," and therefore that method would identify this lesion as suspicious but only if polarized dermatoscopy was employed.

PSWL, which were the critical diagnostic feature in this case, have been attributed to the presence of collagen in the context of dermal fibrosis, which has birefringent properties causing rapid randomization of polarized light thus making the collagen more conspicuous [5]. Dermatopathologic collagen bands are in fact evident in this case (Figures 4 and 5). In attempting to explain the presence of PSWL in melanoma but not nevi, it has been proposed that basic fibroblast growth factor (bFGF), maximally expressed at the advancing front of the neoplasm adjacent to fibrotic changes in the dermis may be associated with tightly woven collagen bundles encircling microinvasive melanoma nests [5] and that these structures may correlate with PSWL. In melanomas, melanocyte-induced de novo collagen 1 type synthesis can be identified with Sirius red staining [5], which has not been demonstrated in nevi [16]. While these factors may play a role in the differential presence of PSWL in melanoma, but not in the associated nevus, the actual dermatopathological correlation remains speculative.

This case illustrates the critical role that polarized dermatoscopy can play and argues strongly for its inclusion in the clinical routine. We also suggest that the observation of polarizing-specific white lines should lead to consideration of excision biopsy in any lesion when either nevus or melanoma is in the differential diagnosis. If polarizing-specific white lines are subtle, they may be best appreciated by comparing the polarized and non-polarized view. For this reason we suggest that both polarizing and non-polarizing modes of dermatoscopy should be employed routinely, this practice being facilitated by dermatoscopes that can be conveniently switched between modes.

\section{References}

1. Bourne P, Rosendahl C, Keir J, Cameron A. BLINCK-A diagnostic algorithm for skin cancer diagnosis combining clinical features with dermatoscopy findings. Dermatol Pract Conc. 2012;2(2):12.

2. Benvenuto-Andrade C, Dusza SW, Agero ALC, et al. Differences between polarized light dermoscopy and immersion contact dermoscopy for the evaluation of skin lesions. Arch Dermatol. 2007;143(3):329-38. 
3. Pan Y, Gareau DS, Scope A, et al. Polarized and nonpolarized dermoscopy: the explanation for the observed differences. Arch Dermatol. 2008;144(6):828-9.

4. Friedman RJ, Rigel DS, Kopf AW. Early detection of malignant melanoma: the role of physician examination and self-examination of the skin. CA Cancer J Clin. 1985;35(3):130-51.

5. Balagula Y, Braun RP, Rabinovitz HS, et al. The significance of crystalline/chrysalis structures in the diagnosis of melanocytic and nonmelanocytic lesions. J Am Acad Dermatol. 2012;67(2):194.e1-8.

6. Vollmer RT. Patient age in Spitz nevus and malignant melanoma: implication of Bayes rule for differential diagnosis. Am J Clin Pathol. 2004;121(6):872-7.

7. Weedon D. Weedon's Skin Pathology, 3rd Edition. Edinburgh: Churchill Livingstone, Elsevier, 2010:4.

8. Pehamberger $\mathrm{H}$, Steiner $\mathrm{A}$, Wolff $\mathrm{K}$. In vivo epiluminescence microscopy of pigmented skin lesions. I. Pattern analysis of pigmented skin lesions. J Am Acad Dermatol. 1987;17(4):571-83.

9. Nachbar F, Stolz W, Merkle T, et al. The ABCD rule of dermatoscopy. High prospective value in the diagnosis of doubtful melanocytic skin lesions. J Am Acad Dermatol. 1994;30(4):551-9.

10. Argenziano G, Soyer HP, Chimenti S, et al. Dermoscopy of pigmented skin lesions: results of a consensus meeting via the Internet. J Am Acad Dermatol. 2003;48(5):679-93.
11. Argenziano G, Fabbrocini G, Carli P, et al. Epiluminescence microscopy for the diagnosis of doubtful melanocytic skin lesions. Comparison of the ABCD rule of dermatoscopy and a new 7-point checklist based on pattern analysis. Arch Dermatol. 1998;134(12):1563-70.

12. Menzies SW, Ingvar C, Crotty KA, McCarthy WH. Frequency and morphologic characteristics of invasive melanomas lacking specific surface microscopic features. Arch Dermatol. 1996;132(10):1178-82.

13. Henning JS, Dusza SW, Wang SQ, et al. The CASH (color, architecture, symmetry, and homogeneity) algorithm for dermoscopy. J Am Acad Dermatol. 2007;56(1):45-52.

14. Rosendahl C, Tschandl P, Cameron A, Kittler H. Diagnostic accuracy of dermatoscopy for melanocytic and nonmelanocytic pigmented lesions. J Am Acad Dermatol. 2011;64(6):1068-73.

15. Rosendahl C, Cameron A, McColl I, Wilkinson D. Dermatoscopy in routine practice-“Chaos and Clues.” Aust Fam Physician. 2012;41(7):482-7.

16. Van Kempen LCLT, Rijntjes J, Mamor-Cornelissen I, et al. Type I collagen expression contributes to angiogenesis and the development of deeply invasive cutaneous melanoma. Int J Cancer. 2008;122(5):1019-29. 